\title{
CONCERNING POLYNOMIALS ON THE UNIT INTERVAL
}

\section{GILBERT LABELLE}

Let $f(x)=\sum_{\nu=0}^{n} a_{\nu} x^{\nu}$ be a polynomial of degree $n$. Given its size on the interval $[-1,1]$ the general problem is to determine how large $\left|f^{(k)}(x)\right|$ can be on the same interval. If we know $\|f\|_{\infty}=\max _{[-1,1]}|f(x)|$ as a measure of the size then a classical theorem of W. Markoff [2] states that

$$
\left\|f^{(k)}\right\|_{\infty} \leqq \frac{n^{2}\left(n^{2}-1^{2}\right)\left(n^{2}-2^{2}\right) \cdots\left(n^{2}-(k-1)^{2}\right)}{1 \cdot 3 \cdot 5 \cdots(2 k-1)}\|f\|_{\infty} .
$$

Here we assume that $\|f\|_{2}=\left(\int_{-1}^{+1}|f(x)|^{2} d x\right)^{1 / 2}$ is given as a measure of the size of $f$ and we obtain a sharp bound for $\left|f^{(k)}(x)\right|$ as well as for the coefficients $a_{k}$. Our result may be stated as follows.

THEOREM. Let $f(x)=\sum_{\nu=0}^{n} a_{\nu} x^{\nu}$ be a polynomial of degree $n$, then for any fixed $k, 0 \leqq k \leqq n$,

$$
\left\|f^{(k)}\right\|_{\infty} \leqq 1 \cdot 3 \cdot 5 \cdots(2 k-1) \cdot \frac{n+k+1}{(2(2 k+1))^{1 / 2}}\left(\begin{array}{c}
n+k \\
n-k
\end{array}\right)\|f\|_{2}
$$

and

(3) $\left|a_{k}\right| \leqq \frac{1 \cdot 3 \cdot 5 \cdots(2 k-1)}{k !}\left(k+\frac{1}{2}\right)^{1 / 2}\left(\begin{array}{c}{[(n-k) / 2]+k+\frac{1}{2}} \\ {[(n-k) / 2]}\end{array}\right)\|f\|_{2}$,

where the symbol $[x]$ denotes as usual the integral part of $x$.

The above inequalities are best possible. Equality in (2) holds only for constant multiples of the polynomial

$$
\sum_{\mu=0}^{n-k}(2 \mu+2 k+1)\left(\begin{array}{c}
2 k+\mu \\
\mu
\end{array}\right) P_{k+\mu}(x) .
$$

Besides, the supremum is attained only at the end points of $[-1,1]$. Equality in (3) is attained only for the constant multiples of the polynomial

$$
\sum_{\mu=0}^{[(n-k) / 2]}(-1)^{\mu}(4 \mu+2 k+1)\left(\begin{array}{c}
k+\mu-\frac{1}{2} \\
\mu
\end{array}\right) P_{k+2 \mu}(x) .
$$

The symbol $P_{\nu}(x)$ in (4) and (5) denotes the Legendre polynomial of degree $\nu$.

Received by the editors September 25, 1967 and, in revised form, November 20, 1967. 
For the proof of the theorem we need a lemma.

Lemma. If $P_{n}(x)$ denotes the Legendre polynomial of degree $n$, then for $0<\theta<\pi, k \geqq 0$ we have

(6) $P_{n}^{(k)}(\cos \theta)=1 \cdot 3 \cdot 5 \cdots(2 k-1) \sum_{\nu=0}^{n} D(k, n-\nu ; \theta) P_{\nu}(\cos \theta)$, where

$$
D(k, \mu ; \theta)=\sum_{\nu_{1}+\cdots+\nu_{k}=\mu} \frac{\sin \nu_{1} \theta \cdots \sin \nu_{k} \theta}{\sin ^{k} \theta} .
$$

We also have

$$
\begin{aligned}
P_{n}^{(k)}(1) & =1 \cdot 3 \cdot 5 \cdots(2 k-1)\left(\begin{array}{l}
n+k \\
n-k
\end{array}\right), \\
P_{n}^{(k)}(-1) & =1 \cdot 3 \cdot 5 \cdots(2 k-1) \cdot(-1)^{n-k}\left(\begin{array}{l}
n+k \\
n-k
\end{array}\right), \\
P_{n}^{(k)}(0) & =1 \cdot 3 \cdot 5 \cdots(2 k-1) \cdot(-1)^{(n-k) / 2}\left(\begin{array}{c}
\frac{n+k-1}{2} \\
\frac{n-k}{2}
\end{array}\right) \\
& =0 \quad \text { if } n \neq k(\bmod 2) .
\end{aligned}
$$

Proof of the lemma. It is well known (see for example [1, p. 365]) that

(11) $\left(1-2 x z+z^{2}\right)^{-1 / 2}=\sum_{n=0}^{\infty} P_{n}(x) z^{n} \quad$ for $-1<x<1,|z|<1$.

Differentiating both sides of (11) $k$ times with respect to $x$, we get

$$
\begin{aligned}
\frac{1 \cdot 3 \cdot 5 \cdots(2 k-1) z^{k}}{\left(1-2 x z+z^{2}\right)^{k}} \cdot \frac{1}{\sqrt{1-2 x z+z^{2}}}= & \sum_{n=0}^{\infty} P_{n}^{(k)}(x) z^{n} \\
& \text { for }-1<x<1,|z|<1 .
\end{aligned}
$$

In other words, for $x=\cos \theta, 0<\theta<\pi$, we have

$$
\frac{1 \cdot 3 \cdot 5 \cdots(2 k-1) z^{k}}{\left[\left(z-e^{i \theta}\right)\left(z-e^{-i \theta}\right)\right]^{k}} \cdot \frac{1}{\sqrt{1-2 z \cos \theta+z^{2}}}=\sum_{n=0}^{\infty} P_{n}^{(k)}(\cos \theta) z^{n}
$$


But

$$
\frac{z}{\left(z-e^{i \theta}\right)\left(z-e^{-i \theta}\right)}=\sum_{\nu=0}^{\infty}\left(\frac{\sin \nu \theta}{\sin \theta}\right) z^{\nu}
$$

so that

$$
\frac{z^{k}}{\left[\left(z-e^{i \theta}\right)\left(z-e^{-i \theta}\right)\right]^{k}}=\left\{\sum_{\nu=0}^{\infty}\left(\frac{\sin \nu \theta}{\sin \theta}\right) z^{\nu}\right\}^{k}=\sum_{\mu=0}^{\infty} D(k, \mu ; \theta) z^{\mu} .
$$

Substituting (13) in (12) we deduce that

$$
\begin{gathered}
1 \cdot 3 \cdot 5 \cdots(2 k-1)\left(\sum_{\mu=0}^{\infty} D(k, \mu ; \theta) z^{\mu}\right)\left(\sum_{\nu=0}^{\infty} P_{\nu}(\cos \theta) z^{\nu}\right) \\
=\sum_{n=0}^{\infty} P_{n}^{(k)}(\cos \theta) z^{n}
\end{gathered}
$$

that is

$$
\begin{gathered}
1 \cdot 3 \cdot 5 \cdots(2 k-1) \sum_{n=0}^{\infty}\left(\sum_{\nu=0}^{n} D(k, n-\nu ; \theta) P_{\nu}(\cos \theta)\right) z^{n} \\
=\sum_{n=0}^{\infty} P_{n}^{(k)}(\cos \theta) z^{n}
\end{gathered}
$$

and (6) follows from equating the coefficients of $z^{n}$ on both sides of this last inequality. Equalities (8), (9), (10) follow easily from standard generating functions.

PROOF OF THE THEOREM. Let

$$
\phi_{\nu}(x)=\left(\nu+\frac{1}{2}\right)^{1 / 2} P_{\nu}(x)
$$

denote the "normalized" Legendre polynomial of degree $\nu$. Then the given polynomial $f(x)$ can be expressed uniquely in the form

$$
f(x)=\sum_{\nu=0}^{n} \alpha_{\nu} \phi_{\nu}(x)
$$

where

$$
\|f\|_{2}=\left(\sum_{\nu=0}^{n} \alpha_{\nu}^{2}\right)^{1 / 2}
$$

Hence, for a fixed $x_{0}\left(=\cos \theta_{0}\right)$ in $[-1,1]$, we get 


$$
\begin{aligned}
\left|f^{(k)}\left(x_{0}\right)\right| & =\left|\sum_{\nu=0}^{n} \alpha_{\nu} \phi_{\nu}^{(k)}\left(\cos \theta_{0}\right)\right| \\
& \leqq\left[\sum_{\nu=0}^{n}\left\{\phi_{\nu}^{(k)}\left(\cos \theta_{0}\right)\right\}^{2}\right]^{1 / 2}\left(\int_{-1}^{+1}|f(x)|^{2} d x\right)^{1 / 2}, \\
& \text { by Schwarz's inequality } \\
& =\left[\sum_{\nu=0}^{n}\left(\nu+\frac{1}{2}\right)\left\{P_{\nu}^{(k)}\left(\cos \theta_{0}\right)\right\}^{2}\right]^{1 / 2}\|f\|_{2} .
\end{aligned}
$$

Equality is attained for and only for the constant multiples of

$$
\sum_{\nu=0}^{n} \phi_{\nu}^{(k)}\left(\cos \theta_{0}\right) \phi_{\nu}(x) .
$$

Now, if $0<\theta_{0}<\pi$, then

$$
\begin{aligned}
\left|D\left(k, \mu ; \theta_{0}\right)\right| & =\left|\sum_{\nu_{1}+\cdots+\nu_{k}=\mu} \frac{\sin \nu_{1} \theta_{0} \cdots \sin \nu_{k} \theta_{0}}{\sin ^{k} \theta_{0}}\right| \\
& \leqq \sum_{\nu_{1}+\cdots+\nu_{k}=\mu}\left|\frac{\sin \nu_{1} \theta_{0}}{\sin \theta_{0}}\right| \cdots\left|\frac{\sin \nu_{k} \theta_{0}}{\sin \theta_{0}}\right| \\
& <\sum_{n_{1}+\cdots+v_{k=\mu}} \nu_{1} \cdots \nu_{k}=D(k, \mu ; 0)=\left(\begin{array}{c}
\mu+k-1 \\
\mu-k
\end{array}\right) .
\end{aligned}
$$

Using this strict inequality in (6) we have

$$
\begin{aligned}
\left|P_{n}^{(k)}\left(\cos \theta_{0}\right)\right| & <\left|P_{n}^{(k)}(1)\right|=1 \cdot 3 \cdot 5 \cdots(2 k-1)\left(\begin{array}{l}
n+k \\
n-k
\end{array}\right) \\
& =\left|P_{n}^{(k)}(-1)\right|,
\end{aligned}
$$

so that the constant factor in (16) will be maximum at $\theta_{0}=0$ and $\theta_{0}=\pi$.

We then conclude that

$$
\begin{aligned}
\left\|f^{(k)}\right\|_{\infty} & \leqq\left[\sum_{\nu=0}^{n}\left(\nu+\frac{1}{2}\right)\left\{P_{\nu}^{(k)}( \pm 1)\right\}^{2}\right]^{1 / 2}\|f\|_{2} \\
& =\frac{1 \cdot 3 \cdot 5 \cdots(2 k-1)}{\sqrt{2}}\left[\sum_{\nu=0}^{n}(2 \nu+1)\left(\begin{array}{l}
\nu+k \\
\nu-k
\end{array}\right)^{2}\right]^{1 / 2}\|f\|_{2} \\
& =\frac{1 \cdot 3 \cdot 5 \cdots(2 k-1)}{\sqrt{2}} \cdot \frac{n+k+1}{(2 k+1)^{1 / 2}}\left(\begin{array}{l}
n+k \\
n-k
\end{array}\right)\|f\|_{2} .
\end{aligned}
$$


To check this summation we proceed as follows. For $n<k$ both (17) and (18) are 0 , for $n=k$ it is easy to see that we also have equality and for $n>k$ put

$$
A_{n}=\frac{(n+k+1)^{2}}{2 k+1}\left(\begin{array}{l}
n+k \\
n-k
\end{array}\right)^{2}
$$

it is easy to check that

$$
A_{n}-A_{n-1}=(2 n+1)\left(\begin{array}{l}
n+k \\
n-k
\end{array}\right)^{2}
$$

which is the last term under the summation sign in (17). This completes the proof of (2). We now prove (3).

The inequality (16) gives for $\theta_{0}=\pi / 2$

$$
\left|f^{(k)}(0)\right| \leqq\left(\sum_{\nu=0}^{n}\left(\nu+\frac{1}{2}\right)\left\{P_{\nu}^{(k)}(0)\right\}^{2}\right)^{1 / 2}\|f\|_{2}
$$

This bound is, by (10), equivalent to

$$
\begin{gathered}
\frac{1 \cdot 3 \cdot 5 \cdots(2 k-1)}{\sqrt{2}}\left(\sum_{\mu=0}^{[(n-k) / 2]}(4 \mu+2 k+1)\left(\begin{array}{c}
\mu+k-\frac{1}{2} \\
\mu
\end{array}\right)^{2}\right)^{1 / 2} \cdot\|f\|_{2} \\
=1 \cdot 3 \cdot 5 \cdots(2 k-1)\left(k+\frac{1}{2}\right)^{1 / 2}\left(\begin{array}{c}
{[(n-k) / 2]+k+\frac{1}{2}} \\
{[(n-k) / 2]}
\end{array}\right) \cdot\|f\|_{2} .
\end{gathered}
$$

To check this last equality we need only to show that

$$
\sum_{\mu=0}^{m}(4 \mu+2 k+1)\left(\begin{array}{c}
\mu+k-\frac{1}{2} \\
\mu
\end{array}\right)^{2}=(2 k+1)\left(\begin{array}{c}
m+k+\frac{1}{2} \\
m
\end{array}\right)^{2} .
$$

For $m=0$ this is true, for $m>0$ put

$$
B_{m}=(2 k+1)\left(\begin{array}{c}
m+k+\frac{1}{2} \\
m
\end{array}\right) \text {. }
$$

It is trivial to verify that

$$
B_{m}-B_{m-1}=(4 m+2 k+1)\left(\begin{array}{c}
m+k-\frac{1}{2} \\
m
\end{array}\right)^{2},
$$

whence the result follows.

The extremal polynomials (4) and (5) are obtained on substituting $\theta_{0}=0, \theta_{0}=\pi, \theta_{0}=\pi / 2$ in (16') and then using (8), (9) and (10).

Our theorem is then completely proved.

Note that 
(i) The multiplying factor in (2) is $O\left(n^{2 k+1}\right)$ as $n \rightarrow \infty$.

(ii) The multiplying factor in (3) is $O\left(n^{k+1 / 2}\right)$ as $n \rightarrow \infty$.

We conclude by raising the following question.

What is the smallest number $L=L(k, p, n, e, q)$ such that

$$
\left\|f^{(k)}\right\|_{p} \leqq L\left\|f^{(e)}\right\|_{q}, \quad 1 \leqq p, q \leqq \infty,
$$

holds for every polynomial $f(x)$ of degree $n$ ?

\section{REFERENCES}

1. P. J. Davis, Interpolation and approximation, Blaisdell, New York, 1963.

2. W. Markoff, Über Polynome, die in einen gegebenen Intervalle möglichst wenig von Null abweichen, Math. Ann. 77 (1916), 213-258.

Université DE Montréal 